\title{
Anomalous Loss of DT Alpha Particles in the Tokamak Fusion Test Reactor
}

\author{
Hans W. Herrmann
}

\author{
A DISSERTATION \\ PRESENTED TO THE FACULTY \\ OF PRINCETON UNIVERSITY \\ IN CANDIDACY FOR THE DEGREE \\ OF DOCTOR OF PHILOSOPHY
}

RECOMMENDED FOR ACCEPTANCE

BY THE DEPARTMENT OF

ASTROPHYSICAL SCIENCES

JUNE, 1997 
(C) Copyright 1997 by Hans W. Herrmann.

All rights reserved. 


\section{Abstract}

Princeton's Tokamak Fusion Test Reactor (TFTR) is the first experimental fusion device to routinely use tritium to study the deuterium-tritium (DT) fusion reaction, allowing the first systematic study of DT alpha $(\alpha)$ particles in tokamak plasmas. A crucial aspect of $\alpha$-particle physics is the fraction of alphas that escape from the plasma, particularly since these energetic particles can do severe damage to the first wall of a reactor.

An escaping alpha collector probe has been developed for TFTR's DT phase. Energy distributions of escaping alphas have been determined by measuring the range of $\alpha$-particles implanted into nickel foils located within the alpha collector. Results at 1.0 MA of plasma current are in good agreement with predictions for first orbit alpha loss. Results at 1.8 MA, however, show a significant anomalous loss of partially thermalized alphas (in addition to the expected first orbit loss), which is not observed with the lost alpha scintillator detectors in DT plasmas, but does resemble the anomalous 'delayed' loss seen in DD plasmas. None of the candidate explanations proposed thus far are fully consistent with the anomalous loss observations.

An experiment designed to study the effect of plasma major radius shifts on $\alpha-$ particle loss has led to a better understanding of $\alpha$-particle dynamics in tokamaks. Intuitively, one might suppose that confined marginally passing $\alpha$-particles forced to move toward higher magnetic field during an inward major radius shift (i.e. compression) would mirror and become trapped particles, leading to increased alpha loss.

Such an effect was looked for during the shift experiment, however, no significant changes in alpha loss to the $90^{\circ}$ lost alpha scintillator detector were observed during 
the shifts. It is calculated that the energy gained by an $\alpha$-particle during the inward shift is sufficient to explain this result. However, an unexpected loss of partially thermalized $\alpha$-particles near the passing/trapped boundary was observed to occur between inward and outward shifts at an intermediate value of plasma current (1.4 MA). This anomalous loss feature is not yet understood. 


\section{Acknowledgments}

Very special thanks go to my advisor, Dr. Stewart J. Zweben, who provided endless advice and guidance. Needless to say, without his support this work would not have been possible. Of course there are many others who have contributed to this effort. In particular, I would like to thank Doug Darrow, the other third of the 'Lost Alpha Team', for his valuable insights. Two others, who were very close to this project, but were unfortunate victims of the Great Reduction in Force of '95, were John Timberlake and Janet Felt. John's superb engineering skills were crucial to the completion of the Alpha Collector, and Janet's meticulous programming skills were instrumental in the modeling of the results.

I am greatly indebted to the managerial ranks of TFTR for realizing the importance of graduate student involvement in 'big science'. These leaders of the TFTR project include Kingston Owens, Dave Johnson, Ken Young, Kevin McGuire, Mike Bell and Rich Hawryluk. I am also grateful for the contributions of the physicists of the TFTR team, in particular, Ben LeBlanc, Ed Synakowski, Ken Hill, Rob Budny, Emil Ruskov, Steve Batha, Fred Levinton, Sid Medley, Zuoyang Chang, Mike Zarnstorff and Schwick von Goeler. Of course, the wisdom of several theorists, such as Harry Mynick, Nicolai Gorolenkov, Roscoe White and C. S. Chang, was essential in the understanding of alpha physics. Fellow students Mark Herrmann, John Wright, Steve Smith, Steve Cauffman, Jaeyoung Park and Bob Heeter, helped me work out many of the details as well as providing moral support.

The assistance of Carl Tilson, Steve Williams and the rest of the HP division,

as well as John Collins, Joe Winston and the rest of the TVPS division is greatly appreciated. Their professional attitude and strict adherence to procedure assured 
my safety during many evolutions involving tritium. Further technical support was provided by Greg Lemunyan, Mike Vocaturo, Art Brooks, Mike Diesso, Judy Giarrusso and Doug Loesser, among others. Administrative support was received from Ann McKee, Betty Carey, Terry Greenberg, and most importantly, Barbara Sarfaty.

This work allowed me to form several valuable external collaborations. Special thanks go to Glenn Chong, Spencer Pitcher, Jim Davis, A.A. Haasz of the University of Toronto's Institute for Aerospace Studies, for developing and operating the sample analysis system used to analyze the foils from the Alpha Collector. Glenn's tireless efforts are much appreciated. From this collaboration sprung several others, including Rick Macauly-Newcomb at McMaster University, who performed calibration implants on their Van de Graaff accelerator, and Armando Antoniazzi at Ontario Hydro Technologies, who helped develop the tritium decontamination method used on the foils. Also, Tom Winter of Pennsylvania State University, Wilkes-Barre campus, was extremely generous in performing time consuming atomic cross section computations for this work.

I would like to thank my readers, who have all been mentioned previously, for their valuable suggestions. These were Roscoe White, Dave Johnson, Stewart Zweben and Doug Darrow.

Finally I wish to thank my extended family who were always there for me: most importantly, my wife, Ryn, and much more recently, my son, Charlie, who have been my inspiration; my parents, Karl and Elfi Herrmann, who have always provided the moral (and proof reading) support I needed; my in-laws, Larry and Tudy Hatch, who have provided many vacation opportunities over the years which have allowed us to 'get away from it all'; and the many siblings, siblings-in-law and friends who have believed in me.

This work was supported by US DoE contract number DE-AC02-76-CHO-3073, Canadian Fusion Fuels Technology Project, and Natural Sciences and Engineering Research Council of Canada 
Dedicated to Ryn and Charlie, for all their love and support! 


\section{Contents}

Abstract

1 Introduction and Motivation 1

1.1 Introduction . . . . . . . . . . . . . . . 2

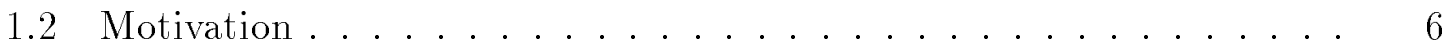

1.3 Objective ........................ 7

1.4 Thesis Outline....................... 7

2 Review of Escaping Alpha Particle Physics in Tokamaks $\quad 8$

2.1 Introduction . . . . . . . . . . . . . 9

2.2 Charged Particle Orbits .................. 9

2.2.1 Single Particle Orbits in Real Space . . . . . . . . . . 9

2.2.2 Orbits in Phase Space ................. 11

2.3 Classical Alpha Loss .................... 14

2.3.1 First Orbit Loss . . . . . . . . . . . 15 
2.3.2 TF Ripple Induced Loss . . . . . . . . . . . . . . 15

2.4 Nonclassical Alpha Loss $\ldots \ldots \ldots \ldots \ldots \ldots$

2.4.1 Collisional Nonprompt Loss _ . . . . . . . . . . . 17

2.4 .2 MHD Induced Loss $\ldots \ldots \ldots \ldots \ldots \ldots$

2.4.3 RF Induced Loss . . . . . . . . . . . . . . . . 19

2.5 Previous Experimental Results . . . . . . . . . . . . . . . 19

2.5.1 Lost Alpha Scintillator Detectors . . . . . . . . . 19

2.5.2 Summary of Previous TFTR Results . . . . . . . . . . 21

3 Alpha Collector - Description $\quad 22$

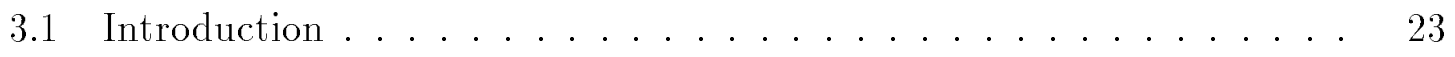

3.2 Detection Technique . . . . . . . . . . . . . . 23

3.3 Probe Head Design . . . . . . . . . . . . . . . 25

3.4 Sample Analysis . . . . . . . . . . . . . . . . . 30

3.4.1 Thermal Desorption Spectrometry . . . . . . . . . . . . 30

3.4 .2 Absolute Calibration . . . . . . . . . . . . . 33

3.4.3 Decontamination . . . . . . . . . . . . . . 34

3.5 TFTR Implementation $\ldots \ldots \ldots \ldots \ldots \ldots \ldots \ldots$

3.6 Comparison to Lost Alpha Scintillator Detectors . . . . . . . . . 40

4 Alpha Collector - Modelling and Results 45 
4.1 Introduction . . . . . . . . . . . . . . . . 46

4.2 First Orbit Loss Model . . . . . . . . . . . . . . . 46

4.2.1 Pitch Angle Distribution _. . . . . . . . . . 46

4.2 .2 Range Distribution ................. 49

4.3 Experimental Results . . . . . . . . . . . . . . . 54

4.3.1 Absolute Fluence .................. 59

4.3.2 Energy Distribution ................ 63

4.3.3 Pitch Angle Distribution . . . . . . . . . . . . . . . 68

4.3.4 Radial Distribution ................ 75

4.3.5 Uncertainties .................. 79

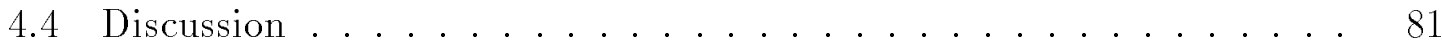

4.4.1 Summary of Experimental Results . . . . . . . . . . 81

4.4.2 Comparison of 1.8 MA Anomalous Loss with Delayed Loss . . 82

4.4.3 Trajectories of anomalous loss orbits .......... 84

4.5 Possible Anomalous Loss Mechanisms . . . . . . . . . . . . 88

4.5.1 Collisional Loss . . . . . . . . . . . . . . . . . 89

4.5.2 Toroidal Field (TF) Ripple Effects . . . . . . . . . . . 89

4.5.3 MHD Effects ....................... 91

4.5.4 Loss of $\mathrm{He}^{+}$from Charge Exchange with Impurities or NBI Neutrals .................... 91

4.5.5 Loss during $I_{p}$ Rampdown . . . . . . . . . . . . 102 
4.5.6 Scattering off RF Limiters and Collimator Walls . . . . . . . 104

4.5.7 Activation of Surrounding Materials . . . . . . . . . . . 104

4.5.8 Foil Surface Fusion .................. 105

4.5.9 Diffusion of Residual He . . . . . . . . . . . . . 105

4.5.10 Diffusion of Implanted He . . . . . . . . . . . . . . 106

4.6 Summary, Conclusions, and Suggestions for Future Work . . . . . 106

$5 \quad$ Major Radius Shift Experiment $\quad 107$

5.1 Introduction . . . . . . . . . . . . . . . . . 108

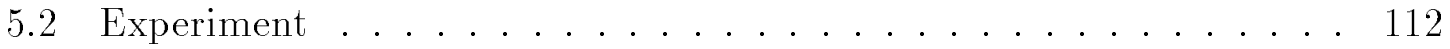

5.2.1 Experimental Design and Initial Expectations . . . . . . . 112

5.2.2 Experimental Results .................. 114

5.3 Discussion ........................... 120

5.3.1 Shift Induced Loss . . . . . . . . . . . . . 120

5.3.2 Anomalous Loss Between the Shifts . . . . . . . . . . . 127

5.4 Summary, Conclusions, and Suggestions for Future Work . . . . . 134

6 Conclusion $\quad 135$

6.1 Introduction . . . . . . . . . . . . . . 136

6.2 Alpha Collector . . . . . . . . . . . . 136

6.2 .1 Summary ...................... 136 


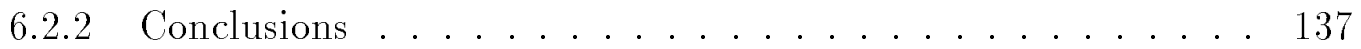

6.2.3 Suggestions for Future Work . . . . . . . . . . . . . 140

6.3 Major Radius Shift Experiment . . . . . . . . . . . . . 140

6.3.1 Summary and Conclusions ............... 140

6.3.2 Suggestions for Future Work . . . . . . . . . . . . . 141

Bibliography 


\section{List of Figures}

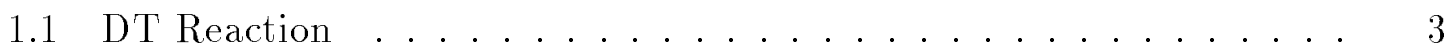

1.2 Tokamak Geometry . . . . . . . . . . . . . . . 5

2.1 Confined Alpha Particle Orbits . . . . . . . . . . 10

2.2 Real and Constants of the motion space $\ldots \ldots \ldots \ldots \ldots$

2.3 Lost Alpha Scintillator Detectors . . . . . . . . . . 20

3.1 Alpha Implantation Ranges in Nickel . . . . . . . . . . . . . 24

3.2 Assembled Probe Heads . . . . . . . . . . . . . . . 25

3.3 Probe Head Blueprint $\ldots \ldots \ldots \ldots \ldots \ldots$

3.4 Foil and Spool Assembly . . . . . . . . . . . . . . 28

3.5 Sample Analysis System . . . . . . . . . . . . . . . . 31

3.6 Calibration Samples . . . . . . . . . . . . . 33

3.7 Alpha Collector shown in Poloidal Cross Section of TFTR . . . . . 36

3.8 Alpha Collector in Top View of TFTR $\ldots \ldots \ldots \ldots \ldots$

3.9 Alpha Collector in Side View of TFTR . . . . . . . . 38 
3.10 Alpha Collector Probe Head Designs . . . . . . . . . . .

4.1 1.8 MA Predicted Alpha Collection Fraction ........... 48

4.2 Alpha Collector Pitch Angle Acceptance ............ 51

4.3 Alpha Implantation Ranges in Nickel . . . . . . . . . . . . 53

4.4 Port transmission fraction vs. Port depth . . . . . . . . . 57

4.51 .0 MA Fluence measurement . . . . . . . . . . . . . . 60

$4.6 \quad 1.8$ MA Fluence measurement . . . . . . . . . . . . . . 61

4.7 Lost Alpha Scintillator Detector $I_{p}$ dependence . . . . . . . . 62

4.81 .0 MA Implantation Range Distributions . . . . . . . . . . 64

$4.9 \quad 1.8$ MA Implantation Range Distributions . . . . . . . . . . 66

4.10 Lost Alpha Scintillator Gyroradius Distributions . . . . . . . . 67

4.11 1.0 MA Left Collection Fraction . . . . . . . . . . . . 69

4.121 .8 MA Left Collection Fraction . . . . . . . . . . . . 71

4.13 1.8 MA Relative Detector Collection Efficiency . . . . . . . . . 72

4.14 Lost Alpha Scintillator Detector Pitch Angle Distributions . . . . . 74

4.15 1.0 MA Alpha Radial Distributions . . . . . . . . . . 76

4.161 .8 MA Alpha Radial Distributions . . . . . . . . . . 78

4.17 1.8 MA Alpha Particle Orbits .............. 85

4.18 Impurity Charge Exchange Cross Sections . . . . . . . . . 93

4.19 Alpha Orbit CX Transition Leading to Outward Transport . . . . . 94 
4.20 Alpha Orbit CX Transition Leading to Inward Transport . . . . . . 96

4.21 1.8 MA Pitch vs. r/a Diagram . . . . . . . . . . . . 97

4.22 Pitch Angle Distribution due to CX . . . . . . . . . . 100

4.23 Energy Distribution due to CX ............... 101

4.24 Slowing Down Times . . . . . . . . . . . . . . . 103

5.1 Marginally Passing $\alpha$-particle Orbit . . . . . . . . . . . . 109

5.2 Collisional Nonprompt Loss . . . . . . . . . . . . . 110

5.3 R Shift Waveforms . . . . . . . . . . . . . 113

5.4 R Shift Lost Alpha Detector Signals . . . . . . . . . . . . . 115

5.5 R Shift Lost Alpha Detector Mean Gyroradius . . . . . . . . . . 117

5.6 R Shift Lost Alpha Detector Mean Pitch Angle . . . . . . . . . . 118

5.7 R Shift Lost Alpha Detector Pitch Angle Distribution . . . . . . . . . 119

$5.8 \mathrm{P} / \mathrm{T}$ Boundary Shift . . . . . . . . . . . . 123

5.9 R Shift Predicted Lost Alpha Detector Signal Increase . . . . . . . 125

5.10 Baseline Mean Gyroradius as a Function of Pitch Angle . . . . . . . . 129

5.11 Calculated Pitch Angle Distributions . . . . . . . . . . . 130

5.12 Nonprompt Loss before and after IN shift . . . . . . . . . . . 132 


\section{List of Tables}

3.1 Comparison of Escaping Alpha Diagnostics . . . . . . . . . . 40

4.1 Alpha Collector Discharge Parameter List . . . . . . . . . 54

4.2 Anomalous Loss Explanations . . . . . . . . . . . 88

5.1 R Shift Shot List . . . . . . . . . . . . . . 114 
Chapter 1

\section{Introduction and Motivation}




\section{$1.1 \quad$ Introduction}

For the past 45 years, scientists have been attempting to realize the potential of fusion energy, the energy source that powers the sun. Fusion could provide a safe, clean, virtually inexhaustible energy source here on earth. Substantial progress has been made in this endeavor, but the goal of commercial fusion power plants has not yet been achieved.

\section{Fusion Reactions}

In a fusion reaction, in which light nuclei are 'fused' together, mass $(\mathrm{m})$ is converted to energy (E) according to Einstein's famous formula $E=m c^{2}$ [1], where the speed of light $\left(\mathrm{c}=3 \times 10^{10} \mathrm{~cm} / \mathrm{s}\right)$ squared provides an enormous conversion factor. The fusion reactions that have been the main focus of fusion energy research are:

$$
\begin{aligned}
\mathrm{D}+\mathrm{T} & \rightarrow{ }^{4} \operatorname{He}(3.52 \mathrm{MeV})+\mathrm{n}(14.1 \mathrm{MeV}) \\
\mathrm{D}+\mathrm{D} & \rightarrow{ }^{3} \mathrm{He}(0.82 \mathrm{MeV})+\mathrm{n}(2.45 \mathrm{MeV}) \\
& \rightarrow \mathrm{T}(1.01 \mathrm{MeV})+\mathrm{H}(3.02 \mathrm{MeV})
\end{aligned}
$$

where the fusion fuel nuclei are the isotopes of hydrogen, deuterium (D) and tritium (T), and the two branches of Eq. 1.2 occur with equal probability. The DT reaction of Eq. 1.1 is illustrated in Fig. 1.1.

In order for the positively charged nuclei on the left sides of Eqs. 1.1 and 1.2 to fuse together they must have sufficient energy to overcome their mutual electrostatic repulsion that is the result of having like electrical charge. Once they get close enough, the strong force, which holds positively charged protons together in a nucleus, takes over and binds them into a new larger nucleus. The resulting nucleus is unstable and immediately decays to the fusion products on the right sides of Eqs. 1.1 and 1.2. The sum of the masses of the products is slightly less than that of the reactants. This mass difference is converted to energy. Conservation of momentum determines the fraction of this energy that each fusion product receives in the form of kinetic energy, which 


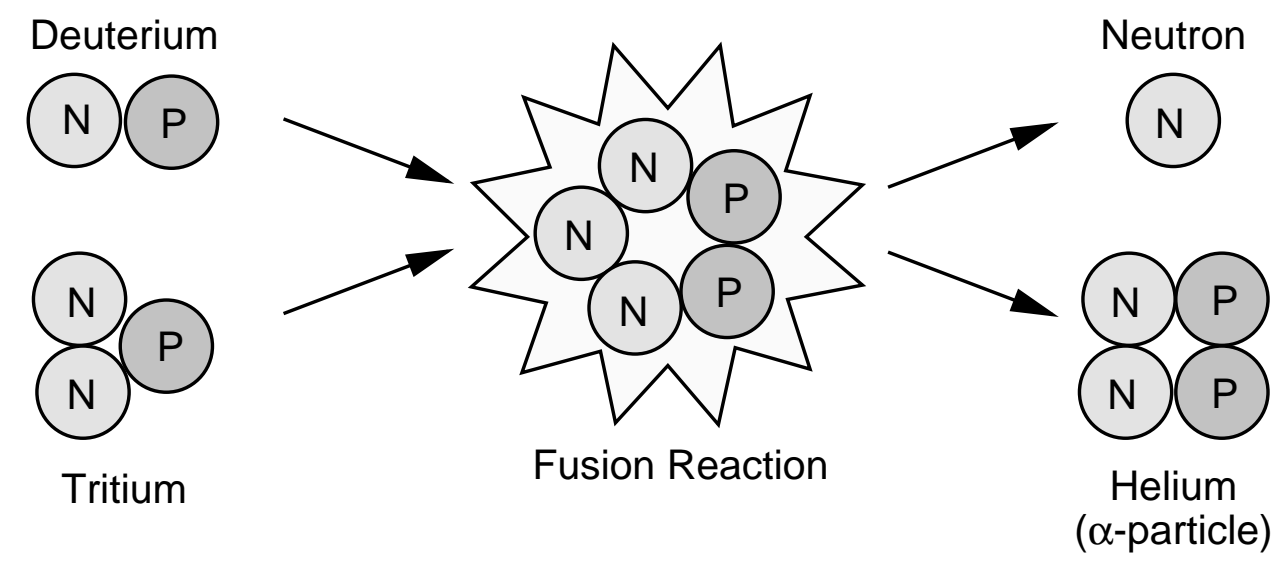

Figure 1.1: The DT fusion reaction of Eq. 1.1 consists of the nuclei of deuterium and tritium fusing together to produce an unstable nucleus which immediately decays to an energetic neutron and helium nucleus (also known as an alpha $(\alpha)$ particle). The nuclei are made up of positively charged protons $(P)$ and electrically neutral neutrons $(N)$.

is shown in parenthesis in Eqs. 1.1 and 1.2 where the units are millions of electron volts $(\mathrm{eV})^{1}$.

\section{Plasma Confinement}

In order for charged particles to have sufficient energy to overcome their repulsion so they can fuse, a working fusion reactor will require the fusion fuel to be heated to hundreds of millions of degrees Celsius $(\gtrsim 10,000 \mathrm{eV})$, more than ten times hotter than the interior of the sun. At these temperatures matter exists in the plasma state, i.e. a gas made up of charged particles (free electrons and ions). The main difficulty in attaining a working fusion reactor has been confining these charged particles and the energy they possess for long enough periods such that the fusion power output exceeds the input power needed to heat the plasma. The point at which the fusion power output is equal to the heating power input is known as 'breakeven'. Princeton's

\footnotetext{
${ }^{1} \mathrm{An} \mathrm{eV}$ is the energy an electron gains when accelerated by a 1 Volt potential. The temperature of a substance is determined by the average kinetic energy of its constituent particles (i.e. molecules, atoms, ions, electrons) and can be expressed in $\mathrm{eV}$, where each $\mathrm{eV}$ is $\approx 11,000^{\circ} \mathrm{C}$.
} 
Tokamak Fusion Test Reactor (TFTR) currently holds the world's record in progress toward breakeven, with an output fusion power about $30 \%$ that of the input power $[2]$.

In magnetic confinement fusion, magnetic fields are used to confine the plasma in a 'magnetic bottle'. This is made possible by the Lorentz force which constrains charged particles to move in helical trajectories about magnetic field lines. This also acts to confine the charged fusion products such as the $3.5 \mathrm{MeV}$ helium (He) nucleus, also known as an alpha $(\alpha)$ particle, seen on the right side of Eq. 1.1. The $14 \mathrm{MeV}$ neutron (n) produced in this reaction, having no charge, is free to pass through the magnetic field and out through the wall of the confinement device where its energy can be used to drive a thermal cycle for the production of electricity. Once the power being transferred to the plasma from the energetic $\alpha$-particles equals the power needed to sustain the fusion reaction, the reactor reaches the self-sustaining point known as 'ignition' at which external heating sources are no longer needed.

\section{Tokamak Reactor}

The confinement device which has been the focus of magnetic fusion research for the past $\sim 30$ years is a Russian invention known as the tokamak [3]. The tokamak has an externally generated toroidal magnetic field $\left(\mathbf{B}_{\mathbf{t}}\right)$ inside a 'doughnut' shaped plasma as shown in Fig. 1.2. What distinguishes the tokamak from other toroidal confinement devices is the addition of a poloidal magnetic field $\left(\mathbf{B}_{\mathbf{p}}\right)$ generated internally by driving a current in the plasma. The resultant magnetic field (B) winds around inside the tokamak in a helical fashion. $\mathbf{B}_{\mathbf{t}}$ is generated by toroidal field (TF) coils that encircle the vacuum vessel (not shown in Fig. 1.2) in the poloidal direction. $\mathbf{B}_{\mathbf{p}}$ is generated by the plasma current $\left(\mathrm{I}_{\text {plasma }}\right)$ which is, in turn, induced through transformer action by a solenoid coil (also not shown) wound around the central axis. The plasma current also acts to heat the plasma through resistive heating similar to the heating of a wire carrying a current. The tokamak plasma is heated further through the injection of energetic fuel atoms from a series of particle accelerators known as neutral beam injection (NBI). 


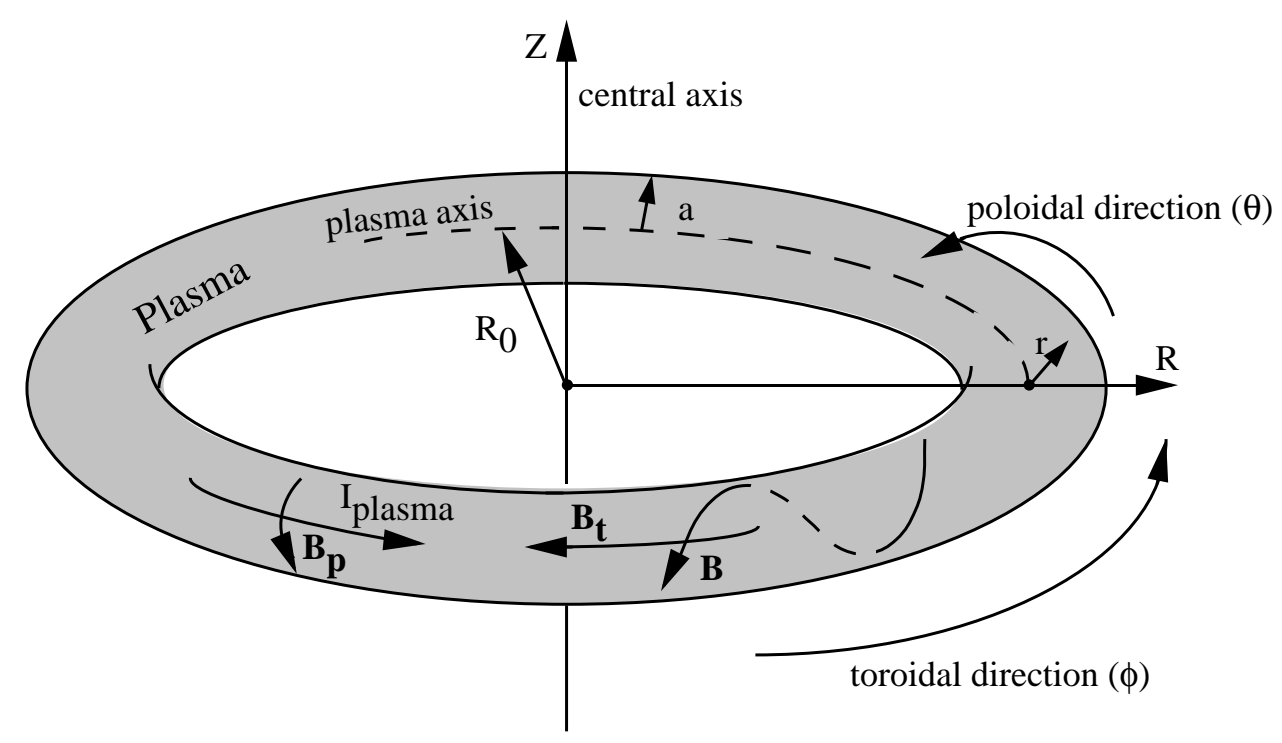

Figure 1.2: The plasma inside a tokamak vacuum vessel is confined by an externally generated toroidal magnetic field $\left(\mathbf{B}_{\mathbf{t}}\right)$ and an internally generated poloidal magnetic field $\left(\mathbf{B}_{\mathbf{p}}\right)$, which combine to form a helical magnetic field (B). The cylindrical $(R, Z, \phi)$ and the toroidal $(r, \theta, \phi)$ coordinate systems are used to describe the toroidal geometry. Plasma dimensions are defined by the major $\left(R_{0}\right)$ and minor (a) radii.

\section{Tritium}

The DD reactions in Eq. 1.2 have been the primary fusion reactions studied in experimental devices. However, it is the DT reaction of Eq. 1.1 that the first generation of commercial fusion reactors will most likely be based on, since it has the highest fusion reaction rate and requires lower plasma temperatures than other fusion reactions. The DT reaction is not normally used in fusion research due to the radioactive concerns involving tritium. Tritium undergoes beta decay, making it a biological hazard if ingested, with a 12.5 year half life. Furthermore, the increased neutron production from the DT reaction activates reactor components such that they too become radioactive. Hence, the introduction of tritium requires special handling and shielding. The DD reaction has provided valuable information regarding the confinement of fusion grade plasmas, but the development of a working fusion reactor requires addressing the unique issues of DT. In particular, alpha particle behavior and its effect on thermonuclear plasmas must be investigated. TFTR is the first experimental 
fusion device to use tritium as a fusion fuel on a routine basis and has conducted the first systematic study of alpha particle physics.

\subsection{Motivation}

\section{Escaping Alpha Particles}

A crucial aspect of alpha particle physics, and the topic of this dissertation, is the fraction of alphas lost to the first wall of the reactor. Alphas which escape from the plasma prior to transferring their excess energy to the plasma (i.e. thermalization) reduce the self-heating power available to achieve ignition. But more importantly, in the design of the International Thermonuclear Experimental Reactor (ITER) and future DT reactors, it will be necessary to predict the alpha particle losses to the first wall and divertor plates, since even a few percent loss may cause damage due to localized heating. Studying alpha particle loss also provides insight into the physics of internal plasma processes, such as plasma instabilities and the effect of numerous alphas acting collectively to perturb the plasma.

Studies of alpha particle loss mechanisms could also prove valuable in developing much needed techniques for improving reactor performance. For instance, once the alphas have thermalized with the bulk plasma, they are no longer useful and actually degrade fusion performance by diluting the plasma. A method of 'helium ash removal' is needed to minimize this dilution by extracting $\alpha$-particles after they have transferred a significant fraction of their energy to the plasma. Another sought after technique is 'alpha channeling' [4]. Normally, the majority of alpha power transfers to the plasma electrons. The electrons do not however take part in the fusion reaction. Reactor efficiency could be greatly enhanced if this power could be diverted directly to the plasma ions, which need energy to fuse. Alpha channeling would use a radio frequency (RF) wave as a catalyst to redirect the alpha power to the bulk ions, or to serve some other useful function such as driving plasma current. 


\section{$1.3 \quad$ Objective}

The main objectives of this thesis were to develop and evaluate a new escaping alpha particle diagnostic, and to conduct new alpha particle experiments on TFTR to gain added insights into alpha particle physics. To this effect, the alpha collector probe, based on the implantation of alpha particles into nickel foils, was developed and implemented on TFTR. This work led to the discovery of an unexpected alpha particle loss, which is not yet understood, but may be a concern in the development of a commercial tokamak reactor. In addition, an experiment designed to study the effect of plasma compression on alpha particle loss has led to a better understanding of alpha particle dynamics in tokamaks.

\subsection{Thesis Outline}

This thesis is organized into six chapters. In chapter 2 there is a review of escaping alpha particle physics in tokamaks including a summary of measurements made on TFTR using scintillator based detectors. Chapter 3 contains a description of the alpha collector, the diagnostic developed to measure escaping alphas in TFTR in the course of this dissertation, including the factors that influenced design choices. Chapter 4 presents the results of escaping alpha measurements made with alpha collector and compares them to numerical modeling and measurements made with the lost alpha scintillator detectors. This analysis reveals the existence of a previously undetected anomalous loss of partially thermalized alphas. Several candidate loss mechanisms are considered, but none of them offer a satisfactory explanation for this anomalous loss. In chapter 5, the results of the major radius shift (i.e. plasma compression) experiment are evaluated with the aid of a powerful 'constants of the motion' theoretical formalism. Chapter 6 contains a summary of results and recommendations for future efforts. 
Chapter 2

Review of Escaping Alpha Particle

Physics in Tokamaks 


\section{$2.1 \quad$ Introduction}

To understand alpha particle loss it is first necessary to understand typical charged particle orbits in tokamaks. Sec. 2.2 contains a description of these orbits for high energy ions. In Secs. 2.3 and 2.4 the various classical and nonclassical high energy ion loss mechanisms are summarized. These loss mechanism descriptions are expanded upon in later chapters as needed. Sec. 2.5 gives a description of the lost alpha scintillator detectors which have provided most of the experimental observations of charged fusion product loss on TFTR and a brief summary of these observations. A comprehensive review of fast ion physics in tokamaks can be found in Ref. [5].

\subsection{Charged Particle Orbits}

\subsubsection{Single Particle Orbits in Real Space}

Charged particles locally follow helical trajectories about magnetic field lines according to the Lorentz force equation:

$$
F=q\left(\mathbf{E}+\frac{1}{c} \mathbf{v} \times \mathbf{B}\right)
$$

where, under most circumstances in a tokamak, the electric field term is negligible for fast ions. As a result of the combination of the toroidal and poloidal magnetic fields, the magnetic field lines in a tokamak spiral around the torus in a helical fashion. In the limit of zero particle energy, the guiding center of charged particle orbits follow these field lines. But for finite energy, vertical drifts associated with the nonuniformity

of the $B$ field play an important role. In particular, the radial dependence $\left(B_{t} \sim \frac{1}{R}\right)$ and curvature of the toroidal magnetic field combine to produce a downward ion drift in TFTR given by:

$$
v_{d r i f t}=\left(E_{\perp}+2 E_{\|}\right) \frac{\mathbf{B} \times \nabla \mathbf{B}}{q B^{2}}
$$




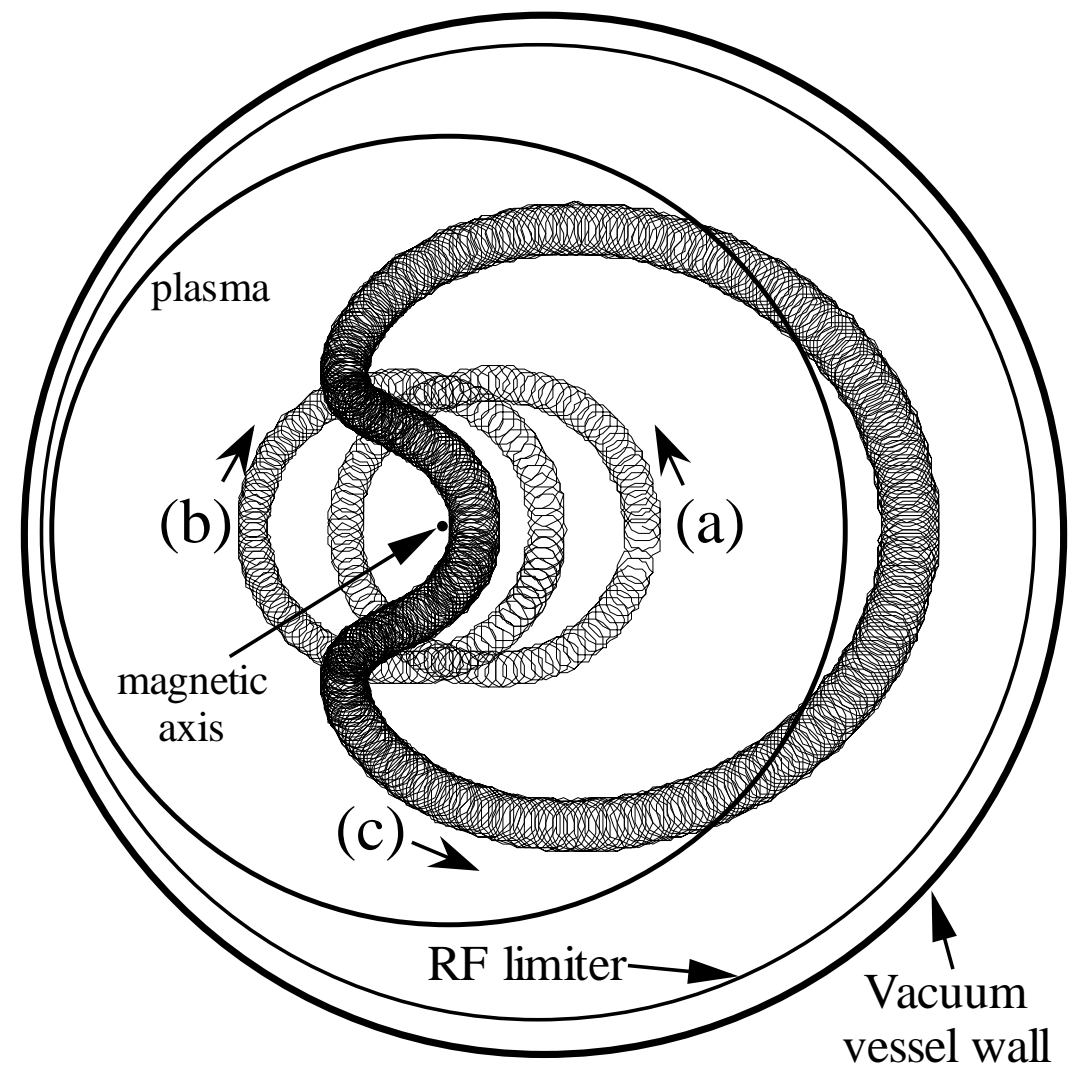

Figure 2.1: Poloidal cross section of TFTR illustrating projections of: (a) a co-going passing orbit; (b) a counter-going passing orbit; and (c) a trapped orbit. Calculated for $3.5 \mathrm{MeV}$ alpha particles in an $R=2.45 \mathrm{~m}, I_{p}=1.6 \mathrm{MA}$ plasma with $B_{t}=5.0$ tesla. Passing orbits (a) and (b) have the same magnetic moment.

where the terms containing the perpendicular and parallel (in relation to the direction of the magnetic field) components of the energy (E) correspond to the $\nabla \mathbf{B}$ drift and the curvature drift, respectively.

If not for the helical spiraling of the magnetic field lines, known as rotational transform, these drifts would cause charged particles to drift down to the bottom of the TFTR vessel. However, the rotational transform causes high energy ions to follow drift surfaces that are displaced outward (inward) in major radius from magnetic flux surfaces for particles that are co-going (counter-going) in relation to the plasma current. Fig. 2.1 shows the poloidal projections representative of the three main orbit 
classifications $^{1}$ : (a) Co-going Passing; (b) Counter-going Passing; and (c) Trapped or 'Banana' orbits. The banana orbit results from the conservation of magnetic moment,

$\mu=\frac{E_{\perp}}{B}$, and energy, $E$. As a charged particle moves into a region of increasing magnetic field, $E_{\perp}$ must increase to maintain $\mu$ constant, while $E_{\|}$must decrease to maintain $E$ constant. When the parallel energy decreases to zero, the particle mirrors and changes its toroidal direction going from counter-going to co-going, or vice versa, forming the banana 'tips'.

\subsubsection{Orbits in Phase Space}

The orbit of a charged particle in a given magnetic configuration is uniquely determined by its instantaneous position, $\mathbf{r}$, and velocity, v, vectors. These quantities define a six dimensional phase space in which the orbit types and loss boundaries can be delineated. However, by tracking only the guiding center motion of a particle, this phase space can be reduced to just three dimensions. The helical Larmor motion about the guiding center can be added back on, if desired, with only a loss of the phase information.

A common method of representing this phase space is by defining three normalized variables at the guiding center's outer midplane crossing point, such as the minor radius, $r / a$, the magnitude of the velocity, $v / v_{0}$ (where $v_{0}$ is the birth velocity), and the pitch, $v_{\|} / v$. However, these quantities are not conserved under most processes that affect charged particle orbits. A better method is to choose three constants of the motion that are either conserved during such processes, or whose changes can be predicted.

Three convenient constants of the motion are the particle's magnetic moment, $\mu$, canonical angular momentum, $P_{\phi}$, and energy, $E[7]$, given by:

$$
\begin{gathered}
\mu=E_{\perp} / B \\
P_{\phi}=m R v_{\phi}-\frac{e}{c} \Psi \cong \frac{m R B_{t} v_{\|}}{B}-\frac{e}{c} \Psi
\end{gathered}
$$

\footnotetext{
${ }^{1}$ For a more complete discussion of particle orbits, including less common orbit types, see Ref. [6].
} 


$$
E=\frac{1}{2} m v^{2}
$$

where $\Psi$ is the poloidal magnetic flux. Solving Eqs. 2.3 and 2.4 for $\mu$ in terms of $P_{\phi}$ we find:

$$
\mu=\frac{E}{B(R, Z)}-\frac{B(R, Z)}{2 m R^{2} B_{t}^{2}(R, Z)}\left(P_{\phi}+\frac{e}{c} \Psi(R, Z)\right)^{2}
$$

It can be seen from Eq. 2.6 that, for a given energy, a fixed $(R, Z)$ position in real space transforms to an inverted parabola in $\left(\mu, P_{\phi}\right)$ space. Thus, the set of all orbits at a given energy passing through one $(R, Z)$ point is a parabola in $\left(\mu, P_{\phi}\right)$ space. The apex of a fixed $(R, Z)$ parabola corresponds to the orbit which has $v_{\|}=0$ at $(R, Z)$, while the right leg of the parabola corresponds to orbits that are co-going $\left(v_{\|}>0\right)$, and the left leg to counter-going $\left(v_{\|}<0\right)$, as they pass through this $(R, Z)$ position. Fig. 2.2(a) defines the three points in real $(R, Z)$ space that make up the plasma boundary in $\left(\mu, P_{\phi}\right)$ space, namely the magnetic axis (MA) and the inner and outer midplane points (IMP and OMP) of the last closed flux surface (LCFS). The parabolas corresponding to these plasma boundary points are shown in Fig. 2.2(b) for fixed $\alpha$-particle energy. All other points in the plasma are represented by parabolas that fall between these three borders, such their apexes fall within the shaded trapped (TL and TC) region.

The different orbit classes are delineated by boundaries in the constants of the motion space as shown in Fig. 2.2(b) ${ }^{2}$. The passing/trapped boundary is formed by the low $\mu$ border of the trapped region which is found by setting $v_{\|}=0$ from the magnetic axis to the LCFS along the inner midplane. Similarly, the high $\mu$ border of the trapped region is found by setting $v_{\|}=0$ from the magnetic axis to the LCFS along the outer midplane. The low $P_{\phi}$ border of the trapped region is found by setting $v_{\|}=0$ along the LCFS from the outer to the inner midplane.

The guiding center trajectory of an $\alpha$-particle orbit can be thought of as a collection of all the fixed $(R, Z)$ parabolas in $\left(\mu, P_{\phi}\right)$ space satisfying Eq. 2.6 that pass through the $\alpha$-particle's fixed $\left(\mu, P_{\phi}\right)$ point, constrained by the condition that the apex of all these parabolas must lie within the shaded trapped region (or to the left of the LCFS $v_{\|}=0$ line for orbits extending outside the LCFS). For example, if an

\footnotetext{
${ }^{2}$ Slight modifications to these borders due to the existence of special orbit classes such as ' $D$ orbits' [7] are ignored for illustrative purposes.
} 
(a)

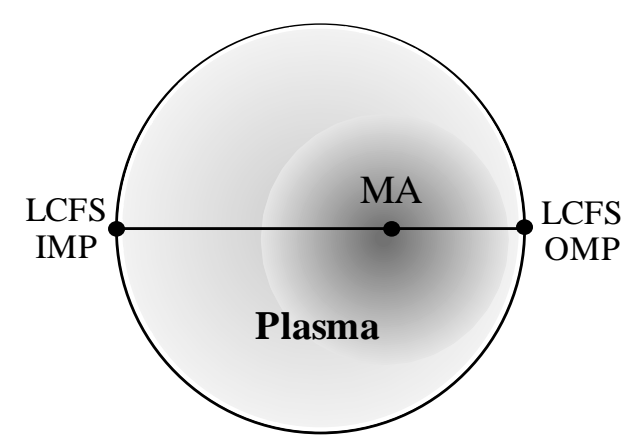

(b)

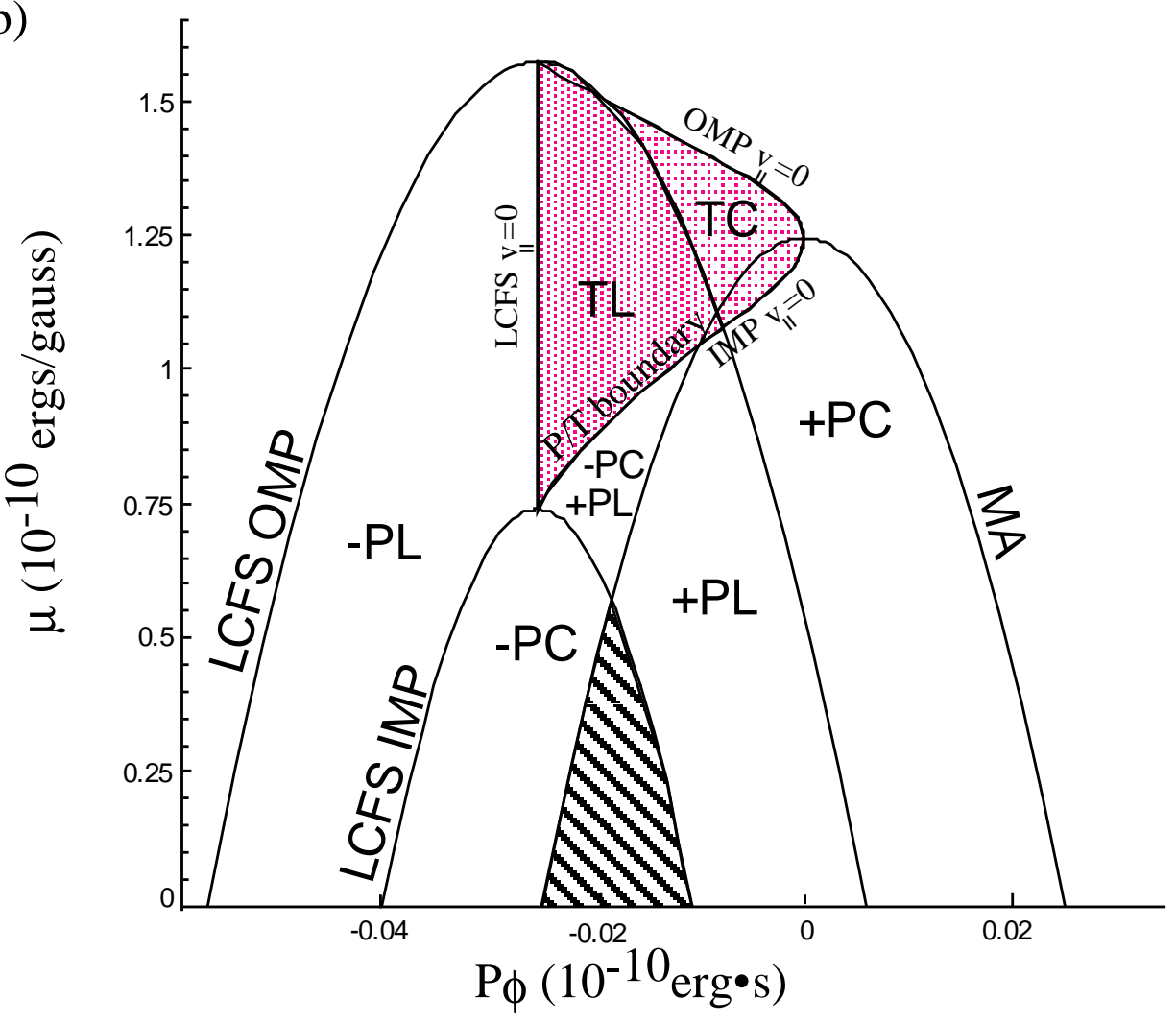

Figure 2.2: (a) Real $(R, Z)$ space defining the plasma magnetic axis (MA) the inner and outer midplane points (IMP and OMP) of the Last Closed Flux Surface (LCFS). (b) $\left(\mu, P_{\phi}\right)$ space calculated for $3.5 \mathrm{MeV} \alpha-$ particles in an $R=2.6 \mathrm{~m}, I_{p}=1.4 \mathrm{MA}$ discharge. The plasma boundary is described by the fixed $(R, Z)$ parabolas corresponding to the points shown in (a). These 3 parabolas, along with the $v_{\|}=0$ along the midplane and LCFS curves, which enclose the shaded trapped particle region, make up the boundaries that delineate orbit types designated by a 3 character code: $+(-)=$ co-(counter-)going; $P(T)=$ passing $($ trapped $) ; C(L)=$ confined $($ lost $)$. 
$\alpha$-particle's $\left(\mu, P_{\phi}\right)$ lies within the trapped region, then the parabola with its apex $\left(v_{\|}=0\right)$ at this $\left(\mu, P_{\phi}\right)$ point is allowed, and the orbit can transition smoothly from being on the counter-going side of a parabola (i.e. left side) to the co-going side and is thus a trapped particle. However, if the $\alpha$-particle's $\left(\mu, P_{\phi}\right)$ lies below the trapped region, then this point is constrained to remain on one side of all the parabolas in the orbit's collection, since the apex of a parabola can not pass through this point, and is thus a passing particle, the direction of which depends on which side of the parabolas the $\left(\mu, P_{\phi}\right)$ point sits on. When the LCFS coincides with the RF limiter radius (e.g. full bore $\mathrm{R}=2.62 \mathrm{~m}$ plasmas in TFTR), an orbit is considered first orbit lost (Sec. 2.3.1) if the apex of any of the parabolas in its collection reach the LCFS $v_{\|}=0$ line. For smaller minor radius plasmas in which the LCFS still intersects the IMP point at the wall (i.e. the bumper limiter in TFTR), the parabola corresponding to the OMP point at the wall defines the new loss boundary for high energy trapped and co-going $\alpha$-particles.

This constants of the motion space provides a convenient description of $\alpha$-particle orbits by allowing all orbits at a given energy to be represented in a two dimensional space. Changes in orbit classifications can be quantified by examining shifts in $\alpha-$ particles' $\left(\mu, P_{\phi}\right)$ or shifts in the orbit classification boundaries themselves due to a change in alpha energy or changes in the magnetic topology. This formalism will be used in the analysis of chapter 5 .

\subsection{Classical Alpha Loss}

Classical losses are determined by the unperturbed magnetic field geometry of the tokamak and can be calculated by following individual particle orbits numerically. Classical losses include first orbit loss, and the TF ripple induced losses. 


\subsubsection{First Orbit Loss}

First orbit loss, the simplest loss, results from particles being born on orbits that intersect the wall on their first bounce (i.e. before completing a poloidal transit). First orbit loss consists of particles born in the loss regions shown in Fig. 2.2 (i.e. + PL, -PL, TL). These particles are lost with very nearly their birth energy since the time for one bounce $(<10 \mu \mathrm{s})$ is much less than the collisional slowing down time $\left(\tau_{s d} \approx 200 \mathrm{~ms}\right.$ energy e-folding time) [8] and are thus also known as 'prompt' loss. Hence, the energy of first orbit lost particles is $\simeq 3.5 \mathrm{MeV}$ with a Doppler spread of up to $\sim \pm 0.5 \mathrm{MeV}$ caused by the beam-target and beam-beam reactions $[9,5]$.

This prompt loss follows the neutron source rate very closely in time since for each neutron produced by fusion, an $\alpha$-particle is also produced. Escaping alpha flux levels can be calculated from a knowledge of the current (or equivalently, q) and fusion source profiles. The global fraction of particles that are first orbit lost decreases with increasing plasma current. This is due to the reduced banana widths of trapped particles in a magnetic geometry with higher rotational transform and hence less overall drift, resulting in a particle staying closer to a given flux surface and thus farther from the walls. Global losses in TFTR can vary from $3 \%$ of the total source rate at $I_{p}=2.7 \mathrm{MA}$ to $\sim 50 \%$ at $I_{p}=0.6 \mathrm{MA}[10]$.

\subsubsection{TF Ripple Induced Loss}

The need for access to the plasma for diagnostics and external heating and fueling sources, such as NBI, necessitates the use of a finite number of discrete toroidal field (TF) coils. TFTR has 20 such TF coils. This results in a rippling of the toroidal field that is most pronounced at the outer midplane. This ripple is responsible for two types of alpha loss, ripple well trapping and stochastic ripple diffusion. 


\section{Ripple Well Trapping}

Ripple well trapping occurs when particles become mirror trapped in the magnetic ripple wells that form between TF coils [11]. These particles are quickly lost to the first wall because the vertical drift is not compensated by the rotational transform of the magnetic field lines. Their orbits, known as 'superbananas', have a pitch angle

$\left(\chi=\cos ^{-1} v_{\|} / v\right)$ very close to $90^{\circ}$ and drift vertically downward only $\sim 0.1 \mathrm{~cm}$ per gyro-orbit. This makes their detection difficult, requiring a collection aperture very close to the tip of a probe to avoid scraping them off on the probe itself. For this reason, this type of loss has not been experimentally verified in TFTR. It is, however, expected to be a small loss channel, since the alpha source rate is small at the outer midplane where the ripple is most pronounced, and only a very small fraction of the alphas in this region occupy the portion of phase space corresponding to ripple trapping. It can, however, be coupled to other loss mechanisms, such as TAE induced loss (Sec. 2.4.2), causing it to be 'fed' to produce enhanced losses.

\section{Stochastic Ripple Diffusion}

Stochastic ripple diffusion (SRD) [12] is a process by which trapped particles, with their banana tips within a threshold region, can diffuse to the wall due to stochasticity brought on by the TF ripple. Passing orbits average out the magnetic field line perturbations brought on by the TF ripple. Trapped particles, however, only sample a portion of the TF ripple perturbation near their banana tips. Stochasticity is then brought on by the toroidal drift that banana orbits experience (or by collisions) which acts to randomize the amount of ripple perturbation sampled at each banana tip. The result is a random jump near each banana tip in the vertical direction to conserve $\mu$. The step size in TFTR is generally on the order of $\sim 1 \mathrm{~cm}$, resulting in a loss process that is relatively fast compared to slowing down, so that these particles are lost with nearly all of their birth energy. This loss process corresponds to trapped confined (TC) particles crossing the trapped confined/lost boundary (i.e. the portion of the OMP LCFS parabola in the trapped region of Fig. 2.2) to the left as the particles' $P_{\phi}$ decreases while $\mu$ and $E$ are conserved. 
As SRD particles diffuse outward in minor radius (from source to sink), the first place they hit the wall is at the outer midplane. For instance, the closet point of approach to the wall for the trapped orbit of Fig. 2.1 is at the midplane. For this reason, SRD loss tends to concentrate within $30^{\circ}$ of the outer midplane. Simulations show that $5 \%-15 \%$ of alphas are lost through SRD at $1.0-2.0$ MA and $R=2.52 \mathrm{~m}$ [13]. SRD is expected to be the dominant classical loss mechanism for alphas at high current in TFTR $\left(I_{p} \geq 1.5 \mathrm{MA}\right)[14]$.

\subsection{Nonclassical Alpha Loss}

Other than classical losses result from varying perturbations such as small scale electric fields (collisions), perturbed magnetic field lines (MHD), and those due to interaction with RF waves.

\subsubsection{Collisional Nonprompt Loss}

Collisional loss results from the pitch angle scattering of marginally passing $\alpha$-particles across the passing/trapped boundary as they slow down, causing these particles to be nonpromptly lost. Normally, the $90^{\circ}$ pitch angle scattering rate is much smaller than the slowing down rate for energetic ions. Particles very close to the passing/trapped boundary, however, only need a small amount of pitch angle change to be scattered into the prompt loss region. Since the small angle pitch angle scattering rate is much larger than the $90^{\circ}$ rate, these particles can scatter across the boundary in the process of slowing down. Hence, this process establishes a partially depleted boundary layer in phase space near the passing/trapped boundary in which the $\alpha$-particle distribution function decays to zero at the boundary. The slope of this distribution function should lead to a diffusive flux of particles across the boundary. This loss is expected to be small in comparison to the usual first orbit loss. For instance, an analytical calculation of Ref. [15] predicted a collisional loss of $\sim 1.3 \%$ in a $1.65 \mathrm{MA}$ 
TFTR supershot, while a TRANSP [16] calculation, which did not include TF ripple, predicted a $\sim 0.3 \%$ nonprompt loss and a total prompt and nonprompt loss rate of $\sim 10 \%$ using the same parameters.

\subsubsection{MHD Induced Loss}

Magnetohydrodynamic (MHD) instabilities act to perturb magnetic field lines and hence also perturb the $\alpha$-particle orbits passing through them. In general, MHD modes are thought to give $\alpha$-particles a radial kick, causing a decrease in $P_{\phi}$ while conserving $\mu$. This can allow previously confined passing particles to cross the passing/trapped boundary in Fig. 2.2 and become lost on large banana orbits. In a similar fashion, MHD can 'feed' other loss channels, such as SRD. MHD modes of any type, whether pressure driven, current driven, or kinetic, can cause loss [17].

With the introduction of tritium, the possibility of alpha driven collective effects arises. A collective instability requires a sufficient population of fast ions such that the ions themselves drive MHD modes. It has been determined that the observed alpha loss in TFTR does not depend on the fusion power level. This indicates that alpha driven collective instabilities either do not or are too weak to induce alpha particle loss in TFTR [9].

A collective instability, the toroidicity-induced Alfvèn eigen (TAE) mode, has been observed in TFTR to be driven by NBI ions, ICRF H-minority tail ions, and more recently by $\alpha$-particles following the end of NBI [18]. However, none of the alpha loss measurements made during these discharges indicate that these modes induce $\alpha$-particle loss. Simulations have, however, shown that TAE modes can transport energetic ions into the ripple trapping region where they are quickly lost to the bottom of the vessel. It has been determined that this synergistic effect was responsible for a melted weld that resulting in a vacuum leak on TFTR [19]. 


\subsubsection{RF Induced Loss}

Radio frequency (RF) waves can interact with high energy ions to put them onto loss orbits [20]. As is the case with MHD-induced loss, RF acts to push fast ions in phase space into existing loss cones, e.g. first orbit or SRD. RF waves, however, can change all three constants of the motion, $E, \mu$, and $P_{\phi}$ [17]. The interaction between fast ions and RF occurs when the wave-particle resonance condition is satisfied,

$$
\omega_{R F}=\Omega_{f}(R)-k_{\|} v_{\| f}
$$

where $\omega_{R F}$ is the applied rf frequency, $\Omega_{f}(R)$ is the fast ion cyclotron frequency, $k_{\|}$is the parallel wave number, and $v_{\| f}$ is the fast ion parallel velocity. The large value of $v_{\| f f}$ allows fast ions to interact over a much larger resonance region than do thermal ions. RF interactions with $\alpha$-particles may play an important role in 'helium ash removal' and 'alpha channeling' (Sec. 1.1).

\subsection{Previous Experimental Results}

Previous measurements of escaping alphas have been made in TFTR using the lost alpha scintillator detectors. These detectors are briefly summarized in Sec. 2.5.1, and the previous DD and DT results obtained using these detectors are summarized in Sec. 2.5.2 to provide a starting point for this thesis.

\subsubsection{Lost Alpha Scintillator Detectors}

Four scintillator detectors are installed on TFTR to detect fusion product losses to the wall [21, 22]. While these detectors were designed to detect alpha particles, they are also capable of detecting the 'alpha-like' DD fusion products (i.e. $3 \mathrm{MeV}$ proton and $1 \mathrm{MeV}$ triton). These detectors are installed at various poloidal angles below the outer midplane $\left(20,45,60\right.$ and $\left.90^{\circ}\right)$ and are all at the same toroidal angle. 


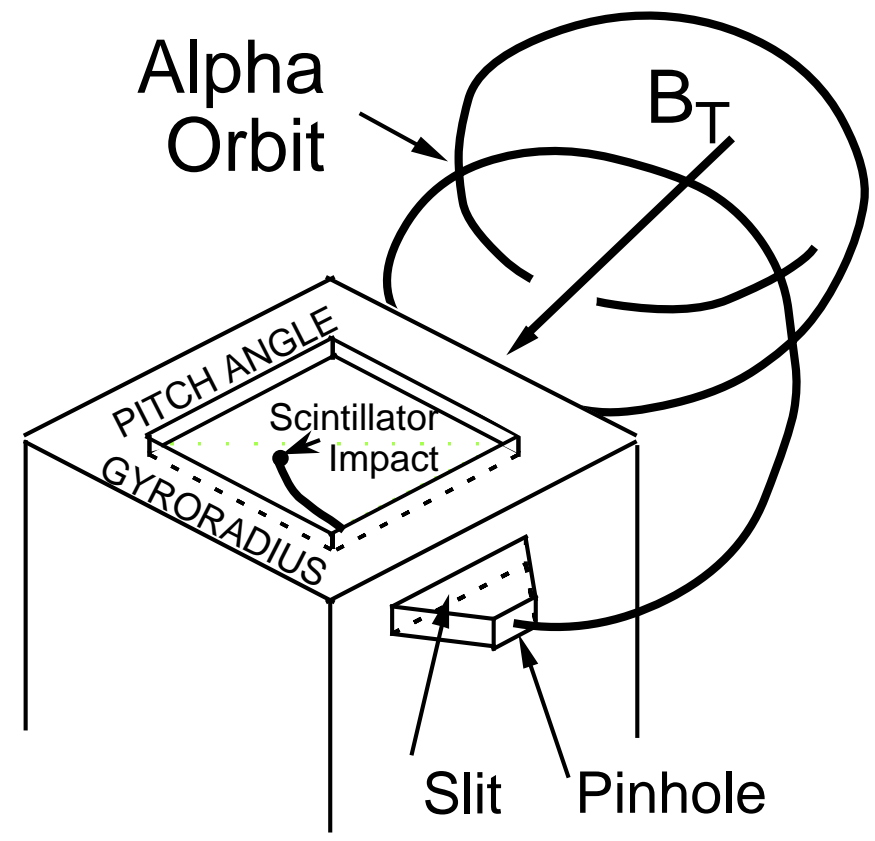

Figure 2.3: Schematic diagram of the lost alpha scintillator detector located $90^{\circ}$ below the outer midplane. The escaping alphas enter a pair of apertures that disperse them in pitch angle and gyroradius. The 2-D image of the visible light emission from the scintillation screen is transmitted through a quartz fibreoptic bundle to a gated intensified video camera for analysis.

As illustrated in Fig. 2.3 each detector consists of a pinhole and slit collimator designed to disperse fusion products along a rectangular scintillator according to gyroradius, $\rho$, (depending on their energy) in one dimension and pitch angle, $\chi$, (depending on their magnetic moment) in the other. The visible light from ion impacts on the scintillator is imaged onto a shielded charged coupled device (CCD) camera and a series of photomultiplier tubes (PMTs). A detector analysis code determines a $(\rho, \chi)$ grid that is used to interpret the camera images. For this grid, the $\rho$ coordinate is the centroid of the predicted scintillator impacts for an ion of gyroradius $\rho_{0}$ (the gyroradius the ion would have if all its energy were put into perpendicular motion, i.e. at $\chi=90^{\circ}$ ) and the $\chi$ coordinate is the orbit's toroidal pitch angle, $\chi_{t}$, measured locally with respect to the co-going toroidal field direction at the detector. Specifics on the design and use of the lost alpha scintillators can be found in Refs. [9, 22]. 


\subsubsection{Summary of Previous TFTR Results}

The escaping alpha measurements contained in this thesis were all made at the bottom of the TFTR vessel (i.e. 90 $)$ in MHD quiescent DT plasmas without RF. All previous observations of alpha loss to the $90^{\circ}$ scintillator detector in MHD quiescent DT plasmas without RF have been consistent with the classical 'single particle' first orbit loss process [9]. There have been no signs of any collective $\alpha$-particle loss processes up to the maximum fusion power level of $10.7 \mathrm{MW}$. The alpha loss to the 45 and $20^{\circ}$ detectors was not consistent with the first orbit loss model alone, perhaps owing to the additional effects of TF ripple induced loss.

Previous measurements of charged fusion product loss to the $90^{\circ}$ scintillator detector in DD plasmas, however, were not fully consistent with just first orbit loss. At small major radii $(\mathrm{R} \leq 2.52 \mathrm{~m})$ and high plasma current $\left(I_{p} \geq 1.4 \mathrm{MA}\right)$ an additional anomalous 'delayed' loss was seen. This loss feature, which is not understood, will be examined in greater detail in Sec. 4.4.2. 